\title{
The Majorant Lyapunov Equation: A Nonnegative Matrix Equation for Robust Stability and Performance of Large Scale Systems
}

\author{
DAVID C. HYLAND AND DENNIS S. BERNSTEIN, MEMBER, IEEE
}

\begin{abstract}
A new robust stability and performance analysis technique is developed. The approach involves replacing the state covariance by its block-norm matrix, i.e., the nonnegative matrix whose elements are the norms of subblocks of the covariance matrix partitioned according to subsystem dynamics. A bound (i.e., majorant) for the block-norm matrix is given by the majorant Lyapunov equation, a Lyapunov-type nonnegative matrix equation. Existence, uniqueness, and computational tractability of solutions to the majorant Lyapunov equation are shown to be completely characterized in terms of $M$ matrices. Two examples are considered. For a damped simple harmonic oscillator with uncertain but constant natural frequency, the majorant Lyapunov equation predicts unconditional stability. And, for a pair of nominally uncoupled oscillators with uncertain coupling, the majorant Lyapunov equation shows that the range of nondestabilizing couplings is proportional to the frequency separation between the oscillators, a result not predictable from quadratic or vector Lyapunov functions.
\end{abstract}

\section{INTRODUCTION}

$\mathrm{T}$ HE importance of robustness in control-system analysis and design cannot be overemphasized. The past ten years' literature reflects considerable frequency-domain development [1]-[11], while recent publications indicate increasing timedomain activity [12]-[19]. Wide variations in underlying assumptions, mathematical settings, and problem data render it difficult, if not impossible, to clearly delineate the relative effectiveness of different methods. Our own philosophical outlook has thus been guided by two general criteria:

1) effectiveness for simple examples;

2) efficiency when applied to large scale problems.

The first criterion involves applying robustness techniques to simple, perhaps trivially obvious, examples to serve as "acid tests." A given method's effectiveness on a collection of such examples can possibly reveal inherent shortcomings. As an illustration of this criterion, consider a damped harmonic oscillator with constant but uncertain natural frequency. Using the notation of [6], stability is guaranteed so long as

$$
\sigma_{\max }\left[R(j \omega)(I+G(j \omega) K(j \omega))^{-1} G(j \omega) L^{-1}(j \omega)\right]<1, \quad \omega \geq 0
$$

where, for $\nu>0$,

$$
G(s)=\left(s^{2}+2 \nu s+\omega_{n}^{2}\right)^{-1}
$$

Manuscript received August 8, 1986; revised May 6, 1987. Paper recommended by Associate Editor, M. G. Safonov. This work was supported in part by the Air Force Office of Scientific Research under Contracts F4962086-C-0002 and F49620-86-C-0038.

The authors are with the Government Aerospace Systems Division, Harris Corporation, Melbourne, FL 32902.

IEEE Log Number 8716541. and uncertainty in the nominal natural frequency $\omega_{n}$ is modeled by

$$
\begin{gathered}
\Delta(s)=L^{-1}(s) \theta(s) R(s)=\delta \omega_{n}^{2}, \\
L(s)=1 / \alpha, \theta(s)=\delta / \alpha, R(s)=\omega_{n}^{2}, K(s)=0, \\
\delta \in[-\min (1, \alpha), \alpha], \quad \alpha>0 .
\end{gathered}
$$

Note that

$$
\sigma_{\max }[\theta(j \omega)] \leq 1, \quad \omega \geq 0
$$

as required in [6]. The perturbation $\Delta(s)$ (modeled as a feedback gain) effectively replaces $\omega_{n}^{2}$ in $G(s)$ by $(1+\delta) \omega_{n}^{2}$. Hence, for a given $\alpha>0$ this uncertainty model permits perturbed natural frequencies in the range $\left[0,(1+\alpha)^{1 / 2} \omega_{n}\right]$. Evaluating (1.1) yields the upper bound

$$
\alpha<\left[\left(\omega_{n}^{2}-\omega^{2}\right)^{2}+4 \nu^{2} \omega^{2}\right]^{1 / 2} / \omega_{n}^{2}, \quad \omega \geq 0
$$

or, equivalently,

$$
\alpha<2 \zeta\left(1-\xi^{2}\right)^{1 / 2}
$$

where $\zeta \triangleq \nu / \omega_{n}$. The conservatism of (1.3) is obviously most pronounced when the damping ratio $\zeta$ is small. In all cases, however, the conservatism is infinite.

The second criterion is obviously subjective and depends upon a variety of factors such as problem structure, designer experience, and computational resources. This criterion is, in our opinion, most important since the need for robustness techniques becomes increasingly critical as system complexity grows. Indeed, the ultimate test of a given approach is to scale it up to larger and larger problems to reveal inherent limitations. Obviously, such tests are not only difficult, but may entail a significant commitment of human and financial resources. Nevertheless, crude predictions are sometimes available, and a case in point is the "curse of dimensionality" encountered in the approach of [9]. Another example involves computational difficulties in obtaining bounds for the $\mu$-function with more than three blocks [10].

The contribution of the present paper is a new robustness analysis method developed specifically for large scale systems. The basic idea, motivated by the work of Siljak [30] on connective stability, is as follows. The system is assumed to be in the form of a collection of subsystems with uncertain local dynamics and uncertain interactions. ${ }^{1}$ Parameter uncertainties are modeled as either structured or unstructured constant variations contained in prescribed sets. The state covariance, partitioned conformably with the subsystem dynamics, is replaced by its block-norm matrix, i.e., the nonnegative matrix each of whose elements is the norm of the corresponding subblock of the original matrix. This nonnegative matrix satisfies a novel inequality designated the

\footnotetext{
1 Uncertainties in a single subsystem can also be regarded as interaction uncertainties. To see this, write $\dot{x}=(A+G) x$ twice so that the uncertainty $G$ is represented by $\left[\begin{array}{ll}0 & G \\ G\end{array}\right]$.
} 
covariance block-norm inequality. The existence of a solution to the majorant Lyapunov equation, i.e., the covariance blocknorm inequality interpreted as an equation, yields an element-byelement bound (i.e., majorant) for the covariance block-norm, hence, assuring robust stability and performance. The relevance of this technique to large scale systems stems from the fact that replacing each subblock of the covariance by its norm can significantly reduce the dimension of the problem. Indeed, the dimension of the majorant Lyapunov equation is equal to the number of subsystems which may be significantly less than the dimension of the original system.

To illustrate the above ideas in more detail, consider the covariance equation

$$
0=(\bar{A}+G) Q+Q(\bar{A}+G)^{T}+V
$$

where $\bar{A}$ denotes the nominal dynamics, $G$ denotes uncertainty in $\bar{A}, V$ is the disturbance intensity, and $Q$ is the state covariance. Assuming that $\bar{A}$ is block diagonal with $r$ diagonal blocks leads to the covariance block-norm inequality (see Proposition 4.2)

$$
\mathbb{Q} * Q \leq \leq \mathrm{Q}+Q^{T}+\mathcal{Q} \text {. }
$$

In (1.5), $\mathcal{Q}, \mathcal{Q}, \mathcal{S}$, and $\vartheta$ are $r \times r$ nonnegative matrices, i.e., each element is a nonnegative number. The matrices $\mathcal{Q}$ and $\mathcal{V}$ are formed by taking the Frobenius norm of each subblock of $Q$ and $V$, while each component of $S$ is a given constant which bounds the spectral norm (largest singular value) of the corresponding subblock of the uncertain perturbation $G$. Hence, $\mathrm{S}$ is a majorant for $G$ in the sense of [21]-[23]. Each element of the matrix $Q$ is bounded above by the smallest singular value of the Kronecker sum [24]-[26] of pairs of diagonal blocks of $\bar{A}$. The operation "*" is the Hadamard product [27], [28], and the ordering " $\leq \leq \leq$ " denotes element-by-element comparison, i.e., the ordering induced by the cone of nonnegative matrices [29], [30].

The majorant Lyapunov equation is obtained by replacing the inequality (1.5) by the $r \times r$ nonnegative matrix equation

$$
\mathcal{Q} * \tilde{Q}=\mathcal{Q} \tilde{Q}+\widetilde{Q} \mathcal{G}^{T}+\nabla
$$

A key result (Corollary 5.1) states that

$$
\mathcal{Q} \leq \leq \tilde{Q}
$$

for all stable $\bar{A}+G$. Consequently (see Theorem 5.1 ), the existence of a unique solution to (1.6) leads directly to a guarantee of robust stability over the range specified by $\mathcal{G}$ and to a performance bound involving $\widetilde{\mathcal{Q}}$. Moreover, solutions of (1.6) exist if and only if the $r^{2} \times r^{2}$ matrix

$$
A \triangleq \operatorname{diag}(\operatorname{vec} Q)-G \oplus \mathrm{G}
$$

is an $M$ matrix [29], [30].

Even when the number of subsystems is large, the majorant Lyapunov equation is generally computationally tractable. Specifically, although $A$ is an $r^{2} \times r^{2}$ matrix, no computations whatsoever need to be carried out with matrices of this dimension. Rather, it suffices to solve only the majorant Lyapunov equation (1.6). In this regard we show that $\bar{Q}$ is given by

$$
\tilde{\mathcal{Q}}=\lim _{i \rightarrow \infty} \tilde{\mathcal{Q}}_{i}
$$

where if $G$ has only off-diagonal nonzero blocks the sequence $\left\{\widetilde{\mathcal{Q}}_{i}\right\}$ is generated by

$$
Q * \tilde{Q}_{i+1}=\mathrm{G} \tilde{Q}_{i}+\tilde{Q}_{i} S^{T}+\vartheta, \quad \tilde{Q}_{0}=0
$$

and is monotonically increasing. Furthermore, the convergence of this sequence is equivalent to $A$ being an $M$ matrix so that it is not even necessary to form $A$. Note that $(1.6)$ does not require the development of new solution techniques. Indeed, since (1.10) is a straightforward iteration, (1.6) is even easier to solve than the original Lyapunov equation (1.4).

To illustrate these results we consider two examples. The first example is the damped oscillator already considered in this section. With little effort the majorant Lyapunov equation yields the (obvious) result that the oscillator is stable for all constant natural frequencies. The second example involves a pair of oscillators with known parameters but with uncertain coupling. The majorant Lyapunov equation yields bounds over which stability is guaranteed, and these bounds are compared to the actual stability region as a function of frequency separation. The main result shows that the robustness to uncertain coupling is proportional to the frequency separation. This weak subsystem interaction robustification mechanism is the principal contribution of the majorant theory. This example has immediate application to the problem of vibration control in flexible structures. For this class of problems the open-loop dynamics can be viewed as a collection of uncoupled oscillators which become coupled via feedback and structural uncertainties.

The majorant bound developed in the present paper is quite different from the widely used quadratic Lyapunov function (see, e.g., [12]-[20]). As can readily be shown using the methods of [12], [17]-[20], the quadratic Lyapunov function yields robust stability and performance by replacing (1.4) by

$$
0=\bar{A} \hat{Q}+\hat{Q} \bar{A}^{T}+\Omega(\hat{Q})+V
$$

where $\Omega(\cdot)$ satisfies

$$
G \hat{Q}+\hat{Q} G^{T} \leq \Omega(\hat{Q})
$$

for all variations $G$. It can then be shown that

$$
Q \leq \hat{Q}
$$

where now, in contrast to (1.7), the ordering in (1.13) is defined with respect to the cone of nonnegative-definite matrices. Indeed, the majorant bound may be more closely related to vector Lyapunov functions [30], [31] and the Lyapunov matrix function [32], [33]. It does not appear possible, however, to use these techniques to obtain the majorant results on robustness due to subsystem frequency separation.

The reader will observe that this paper exploits a wide variety of techniques including nonnegative matrices, block norms, matrix majorants, the Hadamard product, the Kronecker sum, and $M$ matrices. Each of these techniques, except majorants, has, however, been previously applied to control problems in numerous instances. In the special case of scalar subblocks, the blocknorm matrix has, moreover, been utilized by Yedavalli [13]-[15] and others for robustness analysis and design. In this case the block norm is known as the matrix modulus. The variety of algebraic structures employed in the present paper should not be surprising since the quest for increasingly refined robustness techniques can be expected to invoke correspondingly refined uncertainty bounds. Related techniques are employed in [11]. Furthermore, nonnegative matrix equations involving $M$ matrices arise naturally in a variety of settings (see, e.g., [38], [39]).

The contents of the paper are as follows. Section II presents notation, definitions, and lemmas for use throughout the paper. In Section III robust stability and performance are defined for the homogeneous and nonhomogeneous systems. Detailed system structure and uncertainty characterization are given in Section IV and the covariance block-norm inequality is derived. Section $\mathrm{V}$ analyzes the majorant Lyapunov equation to obtain a majorant for the steady-state covariance. The main result, Theorem 5.1, guarantees robust stability and provides a performance bound. Finally, the examples appear in Section VI.

\section{Preliminaries}

The following notation will be used throughout. All matrices are assumed to have real entries. 
菅 $\mathbb{R}, \mathbb{R}^{p \times q}, \mathbb{R}^{p}$

$I_{p}, 0_{p \times q}, 0_{p}$

$\oplus, \otimes$

$\operatorname{col}_{i}(Z)$

$\operatorname{vec}(Z)$

$Z_{(i, j)}$

$Z^{T}$

$Z^{-T}$

tr $Z$

$\operatorname{diag}\left(Z_{1}, \cdots, Z_{p}\right)$

block-diag $\left(Z_{1}, \cdots, Z_{p}\right)$ block-diagonal matrix with listed diag-

$\rho(Z)$

asymptotically stable matrix

nonnegative-definite matrix

positive-definite matrix

$Z_{1} \geq Z_{2}$

$Z_{1}>Z_{2}$

nonnegative matrix

positive matrix

$Z_{1} \geq \geq Z_{2}$

$Z_{1} \gg Z_{2}$

$Z^{H I}$

block-norm matrix

majorant

$\|Z\|_{2}$

$\sigma_{i}(Z)$

$\sigma_{\min }(Z), \sigma_{\max }(Z)$

$\lambda_{\max }(Z)$

$\|Z\|_{s}$

$\|Z\|_{F}$

expected value

real numbers, $p \times q$ real matrices, $\mathrm{R}^{p \times 1}$

$p \times p$ identity matrix, $p \times q$ zero matrix, $0_{p \times p}$

Kronecker sum, Kronecker product

[24]-[27]

Hadamard product [27], [28]

$i$ th column of matrix $Z$

$\left[\begin{array}{c}\operatorname{col}_{1}(Z) \\ \vdots \\ \operatorname{col}_{q}(Z)\end{array}\right] \in \mathrm{\beta}^{p q}, Z \in \mathrm{R}^{p \times q}$

$(i, j)$ element of matrix $Z$

transpose of vector or matrix $Z$

$\left(Z^{T}\right)^{-1}$ or $\left(Z^{-1}\right)^{T}$

trace of matrix $Z$

diagonal matrix with listed diagonal elements

onal blocks

spectral radius of $Z$

matrix with eigenvalues in open lefthalf plane

symmetric matrix with nonnegative eigenvalues $(Z \geq 0)$

symmetric matrix with positive eigenvalues $(Z>0)$

$Z_{1}-Z_{2} \geq 0, Z_{1}, Z_{2}$ symmetric

$Z_{1}-Z_{2}>0, Z_{1}, Z_{2}$ symmetric

matrix with nonnegative elements

$(Z \geq \geq 0$ ) [29], [30]

matrix with positive elements $(Z \gg 0)$

$Z_{1}-Z_{2} \geq \geq 0$

$Z_{1}-Z_{2} \gg 0$

Hadamard inverse, $\left(Z^{H I}\right)_{(i, j)} \triangleq$

$\left[Z_{(i, j)}\right]^{-1}, Z \gg 0$

nonnegative matrix each of whose elements is the norm of a corresponding subblock of a given partitioned matrix nonnegative matrix each of whose elements bounds the corresponding element of a block-norm matrix

Euclidean norm of vector $Z$

singular value of matrix $Z$

smallest and largest singular values of matrix $Z$

largest eigenvalue of symmetric matrix $Z$

$\sigma_{\max }(Z)$ (spectral norm induced by $\|\cdot\|_{2}$ )

$$
\begin{aligned}
\left(\operatorname{tr} Z Z^{T}\right)^{1 / 2} & =\left[\sum_{i, j=1}^{p, q} Z_{(i, j)}^{2}\right]^{1 / 2} \\
& =\left[\sum_{i=1}^{p} \sigma_{i}^{2}\right]^{1 / 2}
\end{aligned}
$$

(Frobenius norm [34]).

In subsequent sections we shall exploit the fact that the norms $\|\cdot\|_{2},\|\cdot\|_{s}$, and $\|\cdot\|_{F}$ coincide for vectors. Hence, if $Z \in R^{p}$, then by interpreting $R^{p}=\mathbb{R}^{p \times 1}$ it follows that

$$
\|Z\|_{2}=\|Z\|_{s}=\|Z\|_{F} .
$$

Furthermore, if $Z \in \mathbb{R}^{p \times q}$, then

$$
\|Z\|_{s} \leq\|Z\|_{F}=\|\operatorname{vec} Z\|_{F}=\|\operatorname{vec} Z\|_{2}=\|\operatorname{vec} Z\|_{s} .
$$

Lemma 2.1: If $Z \in \mathbb{R}^{p \times q}$ and $\hat{Z} \in \mathbb{R}^{q \times r}$ then

$$
\sigma_{\min }(Z)\|\hat{Z}\|_{F} \leq\|Z \hat{Z}\|_{F} \leq\|Z\|_{s}\|\hat{Z}\|_{F} .
$$

If, furthermore, $p=q=r Z \geq 0$, and $\hat{Z}$ is symmetric, then

$$
\operatorname{tr} Z \hat{Z} \leq(\operatorname{tr} Z) \lambda_{\max }(\hat{Z}) \leq(\operatorname{tr} Z)\|\hat{Z}\|_{s} .
$$

Proof: Inequality $(2.4)$ can be found in $[35$, p. 263$]$. To prove (2.3), note that when $Z$ is singular the result is immediate. Otherwise, if $p=q$ replace $Z$ and $\hat{Z}$ in (2.4) by $Z^{-1}$ and $Z \hat{Z}$, respectively. The result now follows from $\left[\sigma_{\max }(Z)\right]^{-1}=$ $\sigma_{\min }\left(Z^{-1}\right)$. If $p \neq q$, then related arguments apply. Finally, (2.5) is given in [36].

Recall [30] that a matrix $S \in \mathcal{B}^{r \times r}$ is an $N$ matrix if $S_{(i, j)} \leq 0$, $i, j=1, \cdots, r, i \neq j$. If, in addition, all principal minors of $S$ are positive, then $S$ is an $M$ matrix.

Lemma 2.2: Suppose $S \in \mathbb{R}^{r \times r}$ is an $N$ matrix. Then the following are equivalent:

i) $S$ is an $M$ matrix;

ii) $\operatorname{det} S \neq 0$ and $S^{-1} \geq \geq 0$;

iii) for each $y \in R^{r}, y \geq \geq 0$, there exists a unique $x \in \mathbb{R}^{r}, x$ $\geq \geq 0$, such that $S x=y$;

iv) there exists $x \in R^{\prime}, x \geq \geq 0$, such that $S x>0$;

v) $I_{r} * S \gg 0$ and each diagonal matrix $D \gg I_{r} * S$ satisfies $\rho\left[D^{-1}\left(I_{r} * S-S\right)\right]<1$.

Proof: The equivalence of statements i), ii), iv), and v) follows from $[30$, p. 396]. The implication ii) $\Rightarrow$ iii) is immediate, and iii) $\Rightarrow$ iv) follows by setting $y=\left[\begin{array}{llll}1 & 1 & \cdots & 1\end{array}\right]^{T}$.

Lemma 2.3: Suppose $S \in$ P $^{r \times r}$ is an $M$ matrix and let $\hat{S} \in$ $R^{r \times r}$ be an $N$ matrix such that $S \geq \geq S$. Then $\hat{S}$ is an $M$ matrix. Proof: See [30, p. 400].

\section{Robust Stability and Performance Bounds}

Consider the $n$ th-order homogeneous system ${ }^{2}$

$$
\begin{gathered}
\dot{x}(t)=(A(\theta)+G) x(t), \quad t \in[0, \infty), \\
G \in G \subset \mathbb{R}^{n \times n}, \\
\theta \in \theta \subset \mathbb{\Omega}^{m},
\end{gathered}
$$

where $A: \Theta \rightarrow R^{n \times n}$ is continuous, $\bar{A} \triangleq A(\bar{\theta})$ denotes the known nominal dynamics for $\bar{\theta} \in \theta, \theta$ denotes the unstructured parametric uncertainty in $\vec{A}, G$ denotes the structured parametric uncertainty in $\bar{A}$, and $0 \in \mathrm{G}$ is the nominal value of $G$. We first consider the stability of (3.1) over $\mathrm{G}$ and $\theta$.

Definition 3.1: If $A(\theta)+G$ is asymptotically stable for all $G$ $\in$ and $\theta \in \Theta$, then the homogeneous system (3.1) is robustly stable over $G$ and $\theta$.

Now consider the $n$ th-order nonhomogeneous system

$$
\dot{x}(t)=(A(\theta)+G) x(t)+w(t), \quad t \in[0, \infty)
$$

where $G \in \mathcal{G}, \theta \in \Theta$, and $w(\cdot)$ is white noise with intensity $V \geq$ 0 . For given $G \in G$ and $\theta \in \theta$, the steady-state average quadratic performance is defined by

$$
J(G, \theta) \triangleq \lim \sup \underset{t}{\xi}\left[x^{T}(t) R x(t)\right]
$$

where $R=R^{T} \geq 0$. The system (3.4) may, for example, denote a control system in closed-loop configuration. There is no need in our development, however, to make such distinctions.

In practice, steady-state performance is only of interest when the system is robustly stable. The following result is immediate.

Proposition 3.1: Suppose the system (3.1) is robustly stable

\footnotetext{
${ }^{2}$ Upon first reading the uncertainty represented by (3.3) can be ignored since the principal contribution concerns the treatment of $(3.2)$.
} 
over $\Theta$ and $\theta$. Then for each $G \in \mathrm{S}$ and $\theta \in \theta$,

$$
J(G, \theta)=\operatorname{tr} Q R
$$

where $n \times n$ nonnegative-definite $Q$ is the unique solution to

$$
0=(A(\theta)+G) Q+Q(A(\theta)+G)^{T}+V .
$$

We shall only be concerned with the case in which $G$ and $\theta$ are compact. Since $Q$ is a continuous function of $G$ and $\theta$, we can define the worst-case average steady-state quadratic performance

$$
J_{\max } \triangleq \max _{G \in \hat{G}, \theta \in \theta} J(G, \theta) .
$$

Since it is difficult to determine $J_{\max }$ explicitly, we shall seek upper bounds.

Definition 3.2: If $J_{\max } \leq \hat{\alpha}$, then $\hat{\alpha}$ is a performance bound for the nonhomogeneous system (3.4) over 9 and $\theta$.

\section{System Structure, Uncertainty Characterization, And THE COVARLANCE BLOCK-NoRm INEQUality}

A discussed in Section I, (3.1) and (3.4) are assumed to be in the form of a large scale system with uncoupled local dynamics and uncertain interactions. Hence, with the subsystem partitioning

$$
n=\sum_{i=1}^{r} n_{i}
$$

the local system dynamics $A(\theta)$ can be decomposed into subsystem dynamics according to

$$
A(\theta)=\underset{i=1, \cdots, r}{\text { block-diag }}\left\{A_{i}(\theta)\right\}
$$

where $A_{i}(\theta) \in \operatorname{R}^{n_{i} \times n_{i}}, \theta \in \theta$. For convenience, denote

$$
\bar{A} \triangleq \underset{i=1, \cdots, r}{\text { block-diag }}\left\{\bar{A}_{i}\right\} \text {. }
$$

Accordingly, $R$ is assumed to be of the form

$$
R=\underset{i=1, \cdots, r}{\operatorname{block}-\operatorname{diag}}\left\{R_{i}\right\}
$$

where $R_{i} \in \mathbb{R}^{n_{i} \times n_{i}}, R_{i} \geq 0, i=1, \cdots, r$. The intensity $V$ and steady-state covariance $Q$ satisfying (3.7) are assumed to be conformably partitioned, i.e.,

$$
\begin{array}{ll}
V=\left\{V_{i j}\right\}_{i, j=1}^{r}, & V_{i j} \in \mathbb{R}^{n_{i} \times n_{j},} \\
Q=\left\{Q_{i j}\right\}_{i, j=1}^{r}, & Q_{i j} \in \mathbb{R}^{n_{i} \times n_{j}} .
\end{array}
$$

For notational simplicity define

$$
V_{i} \triangleq V_{i i}, \quad Q_{i} \triangleq Q_{i i}, \quad i=1, \cdots, r .
$$

Taking the Frobenius norm of each subblock of $V$ and $Q$ leads to the $r \times r$ symmetric nonnegative matrices $\nabla$ and $Q$ defined by

$$
\nabla \triangleq\left\{\left\|V_{i j}\right\|_{F}\right\}_{i, j=1}^{r}, \quad Q \triangleq\left\{\left\|Q_{i j}\right\|_{F}\right\}_{i, j=1}^{r} \text {. }
$$

Note that

$$
\|Q\|_{F}=\|Q\|_{F},\|\nabla\|_{F}=\|V\|_{F} .
$$

A few observations concerning the nominal system, i.e., with $G=0$ and $\theta=\bar{\theta}$, are worth noting. If $\bar{A}$ is stable then so is $\bar{A}_{i}, i$ $=1, \cdots, r$, and there exist unique, nonnegative-definite $\hat{Q}_{i}, \hat{P}_{i}$ $\in\left[R^{n_{i} \times n_{i}}, i=1, \cdots, r\right.$, satisfying

$$
\begin{aligned}
& 0=\bar{A}_{i} \hat{Q}_{i}+\hat{Q}_{i} \bar{A}_{i}^{T}+V_{i}, \\
& 0=\bar{A}_{i}^{T} \hat{P}_{i}+\hat{P}_{i} \bar{A}_{i}+R_{i} .
\end{aligned}
$$

Proposition 4.1: Suppose $\bar{A}$ is asymptotically stable. Then the nominal performance $J_{\text {nom }}$ is given by

$$
J_{\mathrm{nom}} \triangleq J(0, \bar{\theta})=\sum_{i=1}^{r} \operatorname{tr} \hat{Q}_{i} R_{i}=\sum_{i=1}^{r} \operatorname{tr} \hat{P}_{i} V_{i} .
$$

Proof: First note that with $G=0$ and $\theta=\bar{\theta}$ the diagonal blocks of $Q$ satisfying (3.7) coincide with $\hat{Q}_{1}, \cdots, \hat{Q}_{r}$. Thus

$$
\begin{aligned}
J(0, \bar{\theta}) & =\sum_{i=1}^{r} \operatorname{tr} \hat{Q}_{i} R_{i} \\
& =\sum_{i=1}^{r}\left(\operatorname{vec} \hat{Q}_{i}\right)^{T} \operatorname{vec} R_{i} \\
& =\sum_{i=1}^{r}\left[\left(\bar{A}_{i} \oplus \bar{A}_{i}\right)^{-1} \operatorname{vec} V_{i}\right]^{T} \operatorname{vec} R_{i} \\
& =\sum_{i=1}^{r}\left(\operatorname{vec} V_{i}\right)^{T}\left(\bar{A}_{i}^{T} \oplus \bar{A}_{i}^{T}\right)^{-1} \operatorname{vec} R_{i} \\
& =\sum_{i=1}^{r}\left(\operatorname{vec} V_{i}\right)^{T} \operatorname{vec} \hat{P}_{i} \\
& =\sum_{i=1}^{r} \operatorname{tr} \hat{P}_{i} V_{i} .
\end{aligned}
$$

The matrices $G \in \mathbb{G}$ are also conformably partitioned so that

$$
G=\left\{G_{i j}\right\}_{i, j=1}, G_{i j} \in \mathbb{R}^{n_{i} \times n_{j}}
$$

and $\mathbb{G}$ is characterized by

$$
G \triangleq\left\{G \in\left[R^{n \times n}: \sigma_{\max }\left(G_{i j}\right) \leq \gamma_{i j}, \quad i, j=1, \cdots, r\right\}\right.
$$

where $\gamma_{i j} \geq 0, i, j=1, \cdots, r$, are given constants. For convenience, define the $r \times r$ nonnegative matrix

$$
G \triangleq\left\{\gamma_{i j}\right\}_{i, j=1}^{r}
$$

The bound $\mathrm{S}$ is a matrix majorant for $G \in \mathrm{G}$ in the sense of [21]-[23].

Remark 4.1: G is compact and convex.

Finally, let symmetric, positive $Q \in \mathbb{R}^{r \times r}$ satisfy

$$
\mathfrak{Q}_{(i, j)} \leq \min _{\theta \in \theta}\left\{\sigma_{\min }\left(A_{j}(\theta) \oplus A_{i}(\theta)\right)\right\}, \quad i, j=1, \cdots, r .
$$

Proposition 4.2: Let $G \in$ B and $\theta \in \theta$ be such that $A(\theta)+$ $G$ is asymptotically stable and let $n \times n Q \geq 0$ satisfy (3.7). Then $\mathcal{Q}$ defined by (4.7) satisfies

$$
Q * Q \leq \leq \mathrm{Q}+Q \mathrm{Q}^{T}+\nabla
$$

or, equivalently,

$$
A \operatorname{vec} Q \leq \leq \operatorname{vec} \nabla
$$

where

$$
A \triangleq[\operatorname{diag}(\operatorname{vec} \alpha)]-S \oplus S \text {. }
$$

Proof: Expanding (3.7) yields

$$
\begin{array}{r}
-\left[A_{i}(\theta) Q_{i j}+Q_{i j} A_{j}^{T}(\theta)\right]=\sum_{k=1}^{r}\left[G_{i k} Q_{k j}+Q_{i k} G_{j k}^{T}\right]+V_{i j}, \\
i, j=1, \cdots, r .
\end{array}
$$


Bounding the right-hand side of (4.19) from above using (2.4) yields for all $G \in$ G

$$
\begin{aligned}
\| \sum_{k=1}^{r}\left[G_{i k} Q_{k j}+Q_{i k} G_{j k}^{T}\right] & +V_{i j} \|_{F} \\
& \leq \sum_{k=1}^{r}\left[\mathcal{G}_{(i, k)} Q_{(k, j)}+Q_{(i, k)} \mathcal{S}_{(j, k)}\right]+\nabla_{(i, j)}
\end{aligned}
$$

while bounding the left-hand side of (4.19) from below using (2.3) implies for all $\theta \in \theta$

$$
\begin{aligned}
\left\|-\left[A_{i}(\theta) Q_{i j}+Q_{i j} A_{j}^{T}(\theta)\right]\right\|_{F} & =\left\|\operatorname{vec}\left(A_{i}(\theta) Q_{i j}+Q_{i j} A_{j}^{T}(\theta)\right)\right\|_{F} \\
& =\left\|\left(A_{i}(\theta) \oplus A_{i}(\theta)\right) \operatorname{vec} Q_{i j}\right\|_{F} \\
& \geq \sigma_{\min }\left(A_{j}(\theta) \oplus A_{i}(\theta)\right)\left\|\operatorname{vec} Q_{i j}\right\|_{F} \\
& =\sigma_{\min }\left(A_{j}(\theta) \oplus A_{i}(\theta)\right) Q_{(i, j)} \\
& \geq Q_{(i, j)} Q_{(i, j)} .
\end{aligned}
$$

Combining the above inequalities yields (4.16).

Remark 4.2: Since $\mathcal{G} \geq \geq 0$, the $r^{2} \times r^{2}$ matrix $A$ is an $N$ matrix [30].

\section{The Majorant Lyapunov Equation}

In this section we interpret (4.16) as an equality rather than an inequality and consider the Lyapunov-type nonnegative matrix equation

$$
\mathfrak{Q} * \tilde{Q}=S \tilde{Q}+\tilde{Q} S^{T}+\mathcal{V}
$$

or, equivalently,

$$
A \operatorname{vec} \widetilde{Q}=\operatorname{vec} \vartheta
$$

Note that since $\mathcal{Q}$ and $\vee$ are symmetric a unique solution of (5.1) is necessarily symmetric.

Proposition 5.l: The following are equivalent:

i) $A$ is an $M$ matrix;

ii) $\operatorname{det} A \neq 0$ and $A^{-1} \geq \geq 0$;

iii) for each $r \times r$ symmetric $\vee \geq \geq 0$ there exists a unique $r$ $\times r \tilde{\mathcal{Q}} \geq \geq 0$ satisfying (5.1);

iv) there exist $r \times r$ symmetric $\vee \gg 0$ and $r \times r$ symmetric $\widetilde{Q}$ $\geq \geq 0$ satisfying (5.1);

v) diag (vec $\mathcal{Q})-\left(I_{r} * \mathcal{S}\right) \oplus\left(I_{r} * \mathcal{G}\right) \gg 0$ and each diagonal matrix $D \geq \geq \operatorname{diag}(\operatorname{vec} Q)-\left(I_{r} * \mathcal{G}\right) \oplus\left(I_{r} * \mathcal{G}\right)$ satisfies

$$
\rho\left(D^{-1}\left[\mathcal{G} \oplus \mathcal{G}-\left(I_{r} * \mathcal{G}\right) \oplus\left(I_{r} * \mathcal{G}\right)\right]\right)<1
$$

vi) for each $r \times r$ symmetric $\tilde{Q}_{0} \geq \geq 0$ and $r \times r$ symmetric $\nabla$ $\geq \geq 0$, the sequence $\left\{\widetilde{\mathcal{Q}}_{i}\right\}_{i=1}^{\infty}$ generated by

$$
\begin{array}{rl}
\mathcal{Q} & * \tilde{\mathcal{Q}}_{i+1}-\left(I_{r} * \mathcal{G}\right) \tilde{\mathcal{Q}}_{i+1}-\tilde{\mathcal{Q}}_{i+1}\left(I_{r} * \mathcal{G}\right) \\
& =\left(\mathcal{G}-I_{r} * \mathcal{G}\right) \tilde{\mathbb{Q}}_{i}+\tilde{\mathcal{Q}}_{i}\left(\mathcal{G}-I_{r} * \mathcal{G}\right)^{T}+\mathfrak{V}, \quad i=0,1, \cdots,
\end{array}
$$

converges;

vii) for each $r \times r$ symmetric $\widetilde{Q}_{0} \geq \geq 0$ there exists $r \times r$ symmetric $\widetilde{V} \gg 0$ such that the sequence $\left\{\tilde{\mathbb{Q}}_{i}\right\}_{i=1}^{\infty}$ generated by (5.4) converges.

Proof: Statements i)-v) are equivalent to i)-v) of Lemma 2.2. Clearly, vi) implies iii), and vii) implies iv). To show v) implies vi) and vii) note that $I_{r^{2}} *(\mathcal{S} \oplus)=\left(I_{r} * \mathcal{S}\right) \oplus\left(I_{r} * \mathcal{S}\right)$ and

$\operatorname{vec}\left(\mathcal{Q} * \tilde{\mathcal{Q}}_{i+1}\right)=[\operatorname{diag}(\operatorname{vec} \mathcal{Q})]$ vec $\tilde{\mathcal{Q}}_{i+1}$.
Thus, $(5.4)$ is equivalent to

vec $\hat{\mathcal{Q}}_{i+1}=\left[\operatorname{diag}(\operatorname{vec} \mathfrak{Q})-\left(I_{r} * \mathcal{G}\right) \oplus\left(I_{r} * \mathcal{G}\right)\right]^{-1}$

- $\left[\mathcal{G} \oplus \mathcal{G}-I_{r^{2}} *(\mathcal{G} \oplus \mathcal{G})\right]$ vec $\bar{Q}_{i}+[\operatorname{diag}(\operatorname{vec} \mathcal{Q})]^{-1}$ vec $\nabla$.

Thus, vi) and vii) follow from v) with $D=\operatorname{diag}($ vec $Q)-\left(I_{r} *\right.$ G) $\oplus\left(I_{r} * \mathcal{G}\right)$.

Since statements i)-vii) depend only upon $\mathfrak{Q}$ and $\subseteq$ we have the following definition inspired by v)-vii).

Definition 5.1: $(\dot{Q}, \mathcal{S})$ is stable if $A$ is an $M$ matrix.

Remark 5.1: When $I_{r} * S=0$, i.e., when the local dynamics have no structured uncertainty, (5.4) simplifies to

$$
Q * \tilde{Q}_{i+1}=\mathcal{G} \tilde{Q}_{i}+\tilde{Q}_{i} S^{T}+\nabla, \quad i=0,1, \cdots,
$$

or, equivalently,

$$
\widetilde{Q}_{i+1}=Q^{H I} *\left(\mathcal{G} \tilde{Q}_{i}+\widetilde{Q}_{i} \mathcal{G}^{T}+\vartheta\right), \quad i=0,1, \cdots .
$$

The following result shows that for zero initial condition, the iterative sequence is monotonic.

Proposition 5.2: Suppose diag (vec $\mathcal{Q})-I_{r^{2}} *(\mathcal{G} \oplus \mathcal{G}) \gg 0$. Then the sequence $\left\{\tilde{\mathcal{Q}}_{i}\right\}_{i=1}^{\infty}$ generated by (5.4) with $\widetilde{\mathcal{Q}}_{0}=0$ and $\mathcal{V}$ $\geq \geq 0$ is monotonically increasing.

Proof: To simplify notation we consider the case mentioned in Remark 5.1. Hence, assume $\mathscr{Q} \rightarrow 0$. Clearly, if $\widetilde{Q}_{0}=0$, then (5.5a) implies that $\widetilde{Q}_{1}=Q^{H I} * \nabla \geq \geq 0$. Hence, $\widetilde{Q}_{1} \geq \geq \widetilde{\mathcal{Q}}_{0}$. Defining $\Delta \tilde{Q}_{i+1} \triangleq \mathscr{Q}_{i+1}-\tilde{Q}_{i}$, (5.5a) yields

$$
\Delta \tilde{\mathbb{Q}}_{i+1}=\mathfrak{Q}^{H I} *\left(\mathcal{S} \Delta \tilde{\mathcal{Q}}_{i}+\Delta \tilde{\mathcal{Q}}_{i} \mathcal{G}^{T}\right) .
$$

Since $\Delta \tilde{Q}_{1} \geq \geq 0$, the result follows from induction.

Remark 5.2: Proposition 5.2 is a particularly useful result in applications and can be utilized as follows. Setting $\widetilde{Q}_{0}=0$, the sequence $\left\{\widetilde{Q}_{i}\right\}$ can be evaluated by a simple numerical procedure. As will be shown in Theorem 5.1 below, each $\tilde{Q}_{i}$ corresponds to a robust performance measure $\hat{\alpha}_{i}$. For practical purposes the increasing sequence $\left\{\hat{\alpha}_{i}\right\}$ can be generated until either convergence is attained (in which case $\hat{\alpha}=\lim _{i \rightarrow \infty} \hat{\alpha}_{i}$ is a robust performance bound) or a maximum permissible performance level is exceeded. In the latter case the question of convergence is irrelevant since the closed-loop system is known to either be unstable for some $G \in \mathbb{G}$ (i.e., $\hat{\alpha}=\infty$ ) or exceed acceptable performance specifications, thereby necessitating system redesign.

We now prove a comparison result for solutions of (5.1).

Lemma 5.1: Assume $(\hat{Q}, \mathcal{S})$ is stable, let $\hat{\mathcal{Q}}, \hat{\mathcal{S}}$ be $r \times r$ nonnegative matrices where $\widehat{\mathbb{Q}}$ is symmetric, and assume that

$$
\hat{a} \leq \leq \hat{\mathrm{Q}}, \quad \hat{\mathrm{S}} \leq \leq \mathrm{G} .
$$

Then $(\hat{\mathcal{Q}}, \hat{\mathrm{S}})$ is stable. Furthermore, let $r \times r$ symmetric $\tilde{\vartheta}$ satisfy

$$
\hat{v} \leq \leq \vee
$$

let $\tilde{\mathcal{Q}}$ be the unique, nonnegative solution to (5.1), and let $\hat{\mathcal{Q}}$ be the unique solution to

$$
\hat{\mathbb{Q}} * \hat{\mathrm{Q}}=\hat{\mathrm{G}} \hat{\mathrm{Q}}+\hat{\mathrm{Q}} \hat{\mathcal{S}}^{T}+\hat{\mathrm{V}}
$$

Then if $\hat{Q} \geq \geq 0$, it follows that

$$
\hat{Q} \leq \leq \tilde{Q} \text {. }
$$

Proof: Since

$$
\widehat{\AA} \triangleq \operatorname{diag}(\operatorname{vec} \hat{\mathbb{Q}})-\hat{\mathcal{G}} \oplus \hat{\mathcal{G}}
$$

is an $N$ matrix, $A$ is an $M$ matrix, and

$$
\hat{A}-\hat{A}=\operatorname{diag}(\operatorname{vec}(\hat{\mathbb{Q}}-\hat{Q}))+(\mathcal{S}-\hat{\mathcal{G}}) \oplus(\mathcal{S}-\hat{S}) \geq \geq 0
$$


it follows from Lemma 2.3 that $\hat{\mathrm{A}}$ is an $M$ matrix, and thus ( $\hat{\mathrm{Q}}, \hat{\mathrm{Q}}$ ) is stable. Next note that (5.1) and (5.8) imply

$\operatorname{vec}(\tilde{Q}-\hat{Q})=A^{-1}(\hat{A}-A) \operatorname{vec} \hat{Q}+\hat{A}^{-1} \operatorname{vec}(\nabla-\hat{\nabla})$.

Since $\hat{A}-A \geq \geq 0, A^{-1} \geq \geq 0$ (see Lemma 2.2), $\nabla-\hat{\nabla} \geq \geq$ 0 , and $\hat{Q} \geq \geq 0$, it follows that (5.9) is satisfied.

Corollary 5.1: Suppose $(\mathcal{Q}, \mathcal{G})$ is stable and let $\widetilde{Q}$ be the unique, nonnegative solution to (5.1). Furthermore, let $G \in$ G and $\theta \in \theta$ be such that $A(\theta)+G$ is asymptotically stable and define $Q$ by (4.7) for $n \times n Q \geq 0$ satisfying (3.7). Then

$$
Q \leq \leq \widetilde{Q} \text {. }
$$

Proof: By Proposition 4.2, $Q$ satisfies the covariance blocknorm inequality (4.16). In the notation of Lemma 5.1 define

$$
\hat{\mathbb{Q}}=\mathfrak{Q}, \hat{\mathrm{G}}=\mathcal{S}, \hat{\nabla}=\mathfrak{Q} * \mathcal{Q}-\left(\mathcal{Q}+Q \mathcal{G}^{T}\right)
$$

so that (5.6) is satisfied and (4.16) implies (5.7). Note that with the notation (5.11), equation (5.8) has the unique solution $\hat{Q}=\mathcal{Q}$ $\geq \geq 0$. Hence (5.9) implies (5.10).

Theorem 5.1: Assume $A$ is asymptotically stable, $\theta$ is continuously arcwise connected, and $(Q, G)$ is stable. Then the homogeneous system (3.1) is robustly stable over 6 and $\theta$, and the nonhomogeneous system (3.4) has the performance bound

$$
\hat{\alpha}=\max _{\theta \in \Theta}\left\{\sum_{i=1}^{r}\left[\operatorname{tr}\left(\hat{Q}_{i}(\theta) R_{i}\right)+2\left(\operatorname{tr} \hat{P}_{i}(\theta)\right)(\mathcal{G} \tilde{Q})_{(i, i)}\right]\right\}
$$

where $n_{i} \times n_{i}$ nonnegative-definite $\hat{Q}_{i}(\theta)$ and $\hat{P}_{i}(\theta)$ satisfy

$$
\begin{gathered}
0=A_{i}(\theta) \hat{Q}_{i}(\theta)+\hat{Q}_{i}(\theta) A_{i}^{T}(\theta)+V_{i}, \\
0=A_{i}^{T}(\theta) \hat{P}_{i}(\theta)+\hat{P}_{i}(\theta) A_{i}(\theta)+R_{i}
\end{gathered}
$$

and $r \times r \tilde{Q}$ is the unique, nonnegative solution to (5.1).

Proof: First note that since robust stability is independent of the disturbances, we can set $V=I_{n}$ for convenience in proving the first result. Hence, suppose (3.1) is not robustly stable. Since Q is convex (see Remark 4.1), $A$ is asymptotically stable, and $\theta$ is continuously arcwise connected, there exist $G_{0} \in$ G and $\hat{\theta}:[0,1]$ $\rightarrow \Theta$ such that $\hat{A}(\mu) \triangleq A(\hat{\theta}(\mu))+\mu G_{0}$ is asymptotically stable for all $\mu \in[0,1)$, and $\hat{A}(1)$ is not asymptotically stable. Define

$$
Q(\mu, t) \triangleq \int_{0}^{t} e^{\bar{A}(\mu) s} e^{\tilde{A}^{T}(\mu) s} d s, \quad t \geq 0, \mu \in[0,1]
$$

which is monotonically increasing in the nonnegative-definite cone with respect to $t$. Clearly, the limit

$$
Q(\mu) \triangleq \lim _{t \rightarrow \infty} Q(\mu, t), \quad \mu \in[0,1)
$$

exists and satisfies

$$
0=\hat{A}(\mu) Q(\mu)+Q(\mu) \hat{A}^{T}(\mu)+I_{n}, \quad \mu \in[0,1) .
$$

Now define $r \times r$ nonnegative symmetric $Q(\mu)$ by

$$
Q(\mu)=\left\{\left\|Q_{i j}(\mu)\right\|_{F}\right\}_{i, j=1}^{r}
$$

where $Q_{i j}(\mu) \in \mathbb{R}^{n_{i} \times n_{j}}$ and $Q(\mu)$ is partitioned as in (4.5). By Corollary 5.1 with $\theta=\hat{\theta}(\mu), G=\mu G_{0}, \mathcal{Q} \triangleq \mathcal{Q}(\mu), \mu \in[0,1)$, and $V=I_{n}$, it follows from (5.10) that

$$
Q(\mu) \leq \leq \bar{Q}, \mu \in[0,1) .
$$

Hence, by (4.8), (5.15) implies

$$
\|Q(\mu)\|_{F}=\|Q(\mu)\|_{F} \leq\|\tilde{Q}\|_{F}, \mu \in[0,1) .
$$

On the other hand, for $\mu \in[0,1)$ it follows that

$$
\begin{aligned}
Q(\mu) & =Q(\mu)-Q(\mu, t)+Q(\mu, t)-Q(1, t)+Q(1, t) \\
& \geq Q(\mu, t)-Q(1, t)+Q(1, t)
\end{aligned}
$$

which implies, for arbitrary $x \in \mathbb{R}^{n}$,

$$
x^{T} Q(\mu) x \geq x^{T}[Q(\mu, t)-Q(1, t)] x+x^{T} Q(1, t) x
$$

Thus, by continuity of $Q(\mu, t)$ in $\mu$,

$$
\lim _{\mu \rightarrow 1} x^{T} Q(\mu) x \geq x^{T} Q(1, t) x, x \in \Omega^{n} .
$$

Now, since $\hat{A}(1)$ is not asymptotically stable and $\left(\hat{A}(1), I_{n}\right)$ is stabilizable, it follows from [37, Proposition $3.2, \mathrm{p} .67]$ that for some $\bar{x} \in \mathbb{R}^{n}$,

$$
\lim _{t \rightarrow \infty} \bar{x}^{T} Q(1, t) \bar{x}=\infty
$$

Thus, by (5.17)

$$
\lim _{\mu \rightarrow 1} \bar{x}^{T} Q(\mu) \bar{x}=\infty
$$

and thus

$$
\lim _{\mu \rightarrow 1}\|Q(\mu)\|_{F}=\infty .
$$

However, (5.18) contradicts (5.16). Hence, (3.1) is robustly stable over $\mathbb{G}$ and $\theta$.

To derive (5.12) note that since $R$ is block diagonal,

$$
J(G, \theta)=\sum_{i=1}^{r} \operatorname{tr} Q_{i} R_{i}=\sum_{i=1}^{r}\left(\operatorname{vec} Q_{i}\right)^{T} \operatorname{vec} R_{i}
$$

where $Q$ satisfies (3.7). Furthermore, (4.19) implies

$\operatorname{vec} Q_{i}=-\left[A_{i}(\theta) \oplus A_{i}(\theta)\right]^{-1}$

$$
\cdot\left[\operatorname{vec} V_{i}+\sum_{k=1}^{r} \operatorname{vec}\left(G_{i k} Q_{k i}+Q_{i k} G_{i k}^{T}\right)\right] \text {. }
$$

Hence, using Lemma 2.1,

$$
\begin{aligned}
J(G, \theta)= & \sum_{i=1}^{r}\left[\operatorname{tr}\left(\hat{Q}_{i}(\theta) R_{i}\right)\right. \\
& \left.+\sum_{k=1}^{r}\left(\operatorname{vec}\left[G_{i k} Q_{k i}+Q_{i k} G_{i k}^{T}\right]\right)^{T} \operatorname{vec} \hat{P}_{i}(\theta)\right] \\
= & \sum_{i=1}^{r}\left[\operatorname{tr}\left(\hat{Q}_{i}(\theta) R_{i}\right)+\sum_{k=1}^{r} \operatorname{tr} \hat{P}_{i}(\theta)\left(G_{i k} Q_{k i}+Q_{i k} G_{i k}^{T}\right)\right] \\
\leq & \sum_{i=1}^{r}\left[\operatorname{tr}\left(\hat{Q}_{i}(\theta) R_{i}\right)\right. \\
& \left.+\sum_{k=1}^{r}\left(\operatorname{tr} \hat{P}_{i}(\theta)\right) \sigma_{\max }\left(G_{i k} Q_{k i}+Q_{i k} G_{i k}^{T}\right)\right] \\
\leq & \sum_{i=1}^{r}\left[\operatorname{tr}\left(\hat{Q}_{i}(\theta) R_{i}\right)+2\left(\operatorname{tr} \hat{P}_{i}(\theta)\right) \sum_{k=1}^{r} \sigma_{\max }\left(G_{i k}\right) \sigma_{\max }\left(Q_{k i}\right)\right] \\
\leq & \sum_{i=1}^{r}\left[\operatorname{tr}\left(\hat{Q}_{i}(\theta) R_{i}\right)+2\left(\operatorname{tr} \hat{P}_{i}(\theta)\right) \sum_{k=1}^{r} \sigma_{\max }\left(G_{i k}\right)\left\|Q_{k i}\right\|_{F}\right.
\end{aligned}
$$




$$
\begin{aligned}
& \leq \sum_{i=1}^{r}\left[\operatorname{tr}\left(\hat{Q}_{i}(\theta) R_{i}\right)+2\left(\operatorname{tr} \hat{P}_{i}(\theta)\right) \sum_{k=1}^{r} \mathcal{G}_{(i, k)} \tilde{Q}_{(k, i)}\right] \\
& =\sum_{i=1}^{r}\left[\operatorname{tr}\left(\hat{Q}_{i}(\theta) R_{i}\right)+2\left(\operatorname{tr} \hat{P}_{i}(\theta)\right)(S \tilde{Q})_{(i, i)}\right]
\end{aligned}
$$

which yields (5.12).

\section{EXAMPLES}

We first confirm that the damped harmonic oscillator is asymptotically stable for all constant frequency perturbations. Hence, let

$$
r=1, n=n_{1}=2
$$

and

$$
\bar{A}=\bar{A}_{1}=\left[\begin{array}{cc}
-\nu & \omega \\
-\omega & -\nu
\end{array}\right]
$$

where $\nu>0$ and $\omega \in \mathbb{R}$. To represent frequency uncertainty let $\mathbb{G}$ $=\{0\}, \theta=[R, \bar{\theta}=0$, and

$$
A(\theta)=\bar{A}+\theta\left[\begin{array}{rr}
0 & 1 \\
-1 & 0
\end{array}\right]
$$

Note that $A(\theta)$ is stable for all $\theta \in \mathbb{R}$ with poles $-\nu \pm j(\omega+\theta)$. Note that $A(\theta)$ can be diagonalized by means of the unitary transformation

$$
\phi=\frac{1}{\sqrt{2}}\left[\begin{array}{cc}
1 & 1 \\
j & -j
\end{array}\right], \quad \phi^{-1}=\frac{1}{\sqrt{2}}\left[\begin{array}{cc}
1 & -j \\
1 & j
\end{array}\right]
$$

so that

$$
\hat{A}(\theta) \triangleq \phi^{-1} A(\theta) \phi=\left[\begin{array}{cc}
-\nu+j(\omega+\theta) & 0 \\
0 & -\nu-j(\omega+\theta)
\end{array}\right] .
$$

Hence, using

$$
A(\theta) \oplus A(\theta)=\left(\phi^{-1} \otimes \phi^{-1}\right)(\hat{A}(\theta) \oplus \hat{A}(\theta))(\phi \otimes \phi)
$$

it follows that

$$
\sigma_{\min }(A(\theta) \oplus A(\theta))=2 \nu, \quad \theta \in \Omega .
$$

Defining [see (4.15)]

$$
Q=Q_{(1,1)}=2 \nu
$$

and $\mathcal{G}=0$, the scalar majorant Lyapunov equation (5.1) has the solution

$$
\tilde{Q}=\vee / 2 \nu
$$

where $\vartheta=\|V\|_{F}$. Choosing $V=I_{2}$ and noting that $A=Q=2 \nu$ $>0$ is an $M$ matrix, Theorem 5.1 guarantees robust stability for all frequency variations $\theta \in$ 踶.

The next example has been chosen to demonstrate the robustness of a pair of nominally uncoupled oscillators with respect to uncertain coupling. Hence, let

$$
n=4, r=2, n_{1}=n_{2}=2
$$

and

$$
\bar{A}_{i}=\left[\begin{array}{cc}
-v & \omega_{i} \\
-\omega_{i} & -\nu
\end{array}\right], \quad i=1,2
$$

where $\nu, \omega_{1}, \omega_{2} \geq 0$. Furthermore, let $\theta=\{\bar{\theta}\}$ and

$$
S=\left[\begin{array}{cc}
0 & \gamma_{12} \\
\gamma_{21} & 0
\end{array}\right]
$$

which denotes the fact that the local subsystem (oscillator) dynamics are assumed to be known. Since

$$
\sigma_{\min }\left(\bar{A}_{j} \oplus \bar{A}_{i}\right)=\left[4 \nu^{2}+\left(\omega_{j}-\omega_{i}\right)^{2}\right]^{1 / 2}
$$

define

$$
Q=\left[\begin{array}{cc}
2 v & {\left[4 \nu^{2}+\left(\omega_{1}-\omega_{2}\right)^{2}\right]^{1 / 2}} \\
{\left[4 \nu^{2}+\left(\omega_{1}-\omega_{2}\right)^{2}\right]^{1 / 2}} & 2 \nu
\end{array}\right] .
$$

Letting $V=I_{4}$ yields $\nabla=2 I_{2}$. Solving (5.1) yields

$$
\begin{gathered}
\tilde{\mathcal{Q}}_{(1,1)}=\left(2 \nu^{2} \hat{\delta}-\gamma_{12} \gamma_{21}+\gamma_{12}^{2}\right) / 2 \sqrt{2} \nu\left(\nu^{2} \hat{\delta}-\gamma_{12} \gamma_{21}\right), \\
\tilde{Q}_{(1,2)}=\left(\gamma_{12}+\gamma_{21}\right) / 2 \sqrt{2}\left(\nu^{2} \delta-\gamma_{12} \gamma_{21}\right), \\
\tilde{Q}_{(2,2)}=\left(2 \nu^{2} \hat{\delta}-\gamma_{12} \gamma_{21}+\gamma_{21}^{2}\right) / 2 \sqrt{2} \nu\left(\nu^{2} \hat{\delta}-\gamma_{12} \gamma_{21}\right)
\end{gathered}
$$

where

$$
\hat{\delta} \triangleq\left[1+\delta^{2}\right]^{1 / 2}, \delta \triangleq\left(\omega_{1}-\omega_{2}\right) / 2 \nu .
$$

Clearly, $\tilde{Q}$ is nonnegative if and only if

$$
\gamma_{12} \gamma_{21}<\nu^{2} \delta \text {. }
$$

The bound (6.1) characterizes the magnitude of coupling uncertainty for which stability is guaranteed. Note that the parameter $\delta$ is a measure of the frequency separation of the oscillators relative to the damping. When $\delta \gg 1,(6.1)$ becomes asymptotically

$$
\gamma_{12} \gamma_{21}<\frac{\nu}{2}\left|\omega_{1}-\omega_{2}\right|
$$

which confirms the intuitive expectation that robust stability is proportional to damping and subsystem frequency separation. This result does not appear to be predictable from quadratic or vector Lyapunov functions.

To evaluate the conservatism inherent in the bound (6.1) we solve for the actual stability region. To render the calculation tractable we assume that $G_{12}$ and $G_{21}$ have the structured form

$$
G_{i j}=\left[\begin{array}{cc}
\alpha_{i j} & \beta_{i j} \\
-\beta_{i j} & \alpha_{i j}
\end{array}\right] \text {. }
$$

By constraining (6.3) the set of coupling variations is reduced, which may or may not lead to a larger stability region. Thus, our estimate of conservatism may itself be conservative, i.e., the actual conservatism may indeed be less than the following analysis indicates. However, without (6.3) the development becomes intractable. This calculation will thus be called semiexact.

By considering the characteristic equation for $\bar{A}+G$, lengthy manipulation shows that $\bar{A}+G$ is stable if and only if

$$
\gamma_{12} \gamma_{21}<2 \nu^{2}\left(-\epsilon+\left[1+\delta^{2}\left(1-\epsilon^{2}\right)\right]^{1 / 2}\right) /\left(1-\epsilon^{2}\right)
$$

where $\epsilon \in(0,1]$ is the smallest positive real root of

$$
\epsilon=\left(1+\epsilon^{2}\right)\left[1+\delta^{2}\left(1-\epsilon^{2}\right)\right]^{1 / 2} /\left[2+\delta^{2}\left(1-\epsilon^{2}\right)\right] .
$$

The majorant bound (6.1) and semiexact bound (6.4) are illustrated in unified form in Fig. 1. For $\delta \gg 1$ note that $\epsilon=$ $O\left(\delta^{-1}\right)$ and thus (6.4) becomes asymptotically

$$
\gamma_{12} \gamma_{21}<\nu\left|\omega_{1}-\omega_{2}\right|
$$




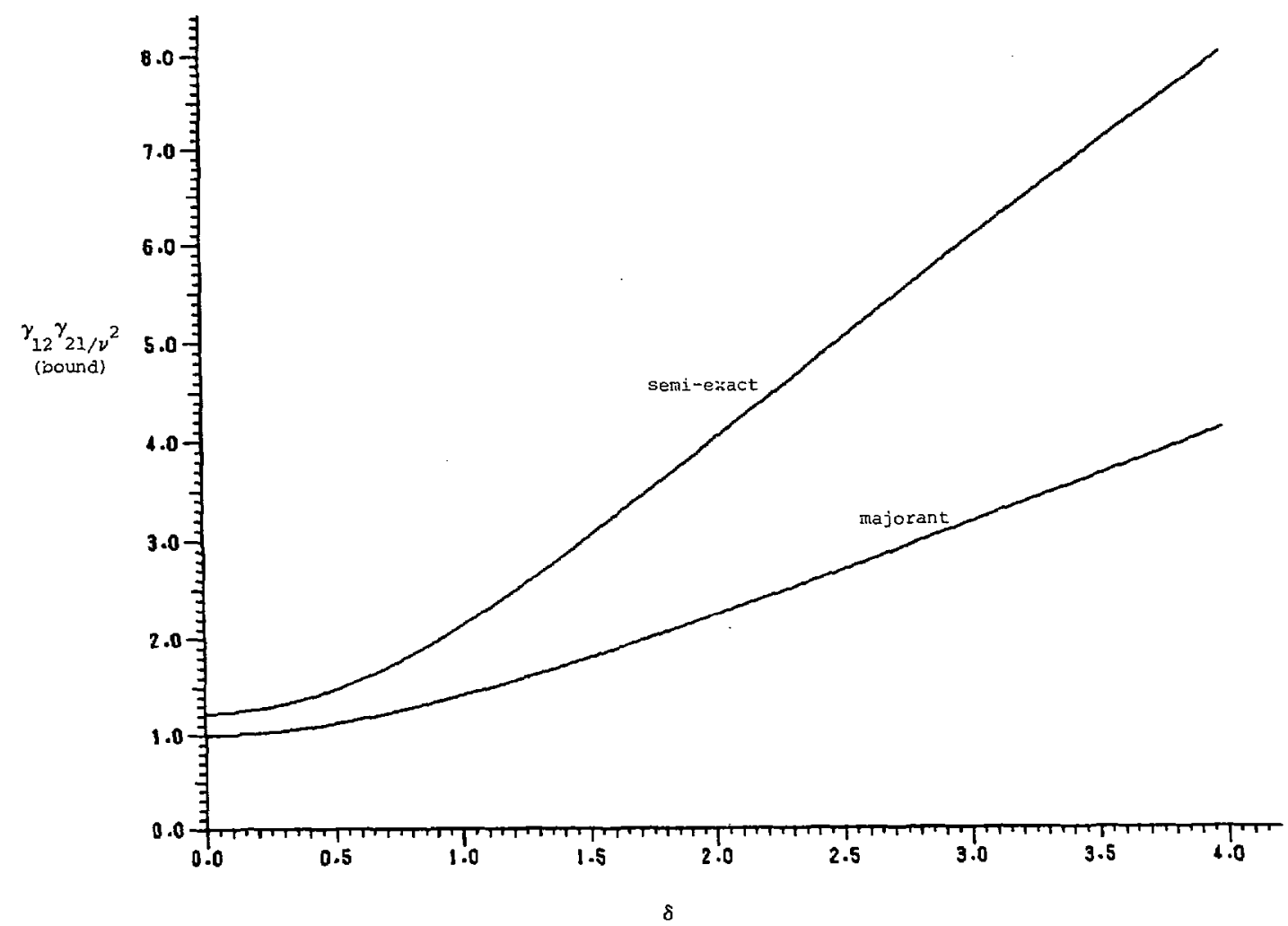

Fig. 1 .

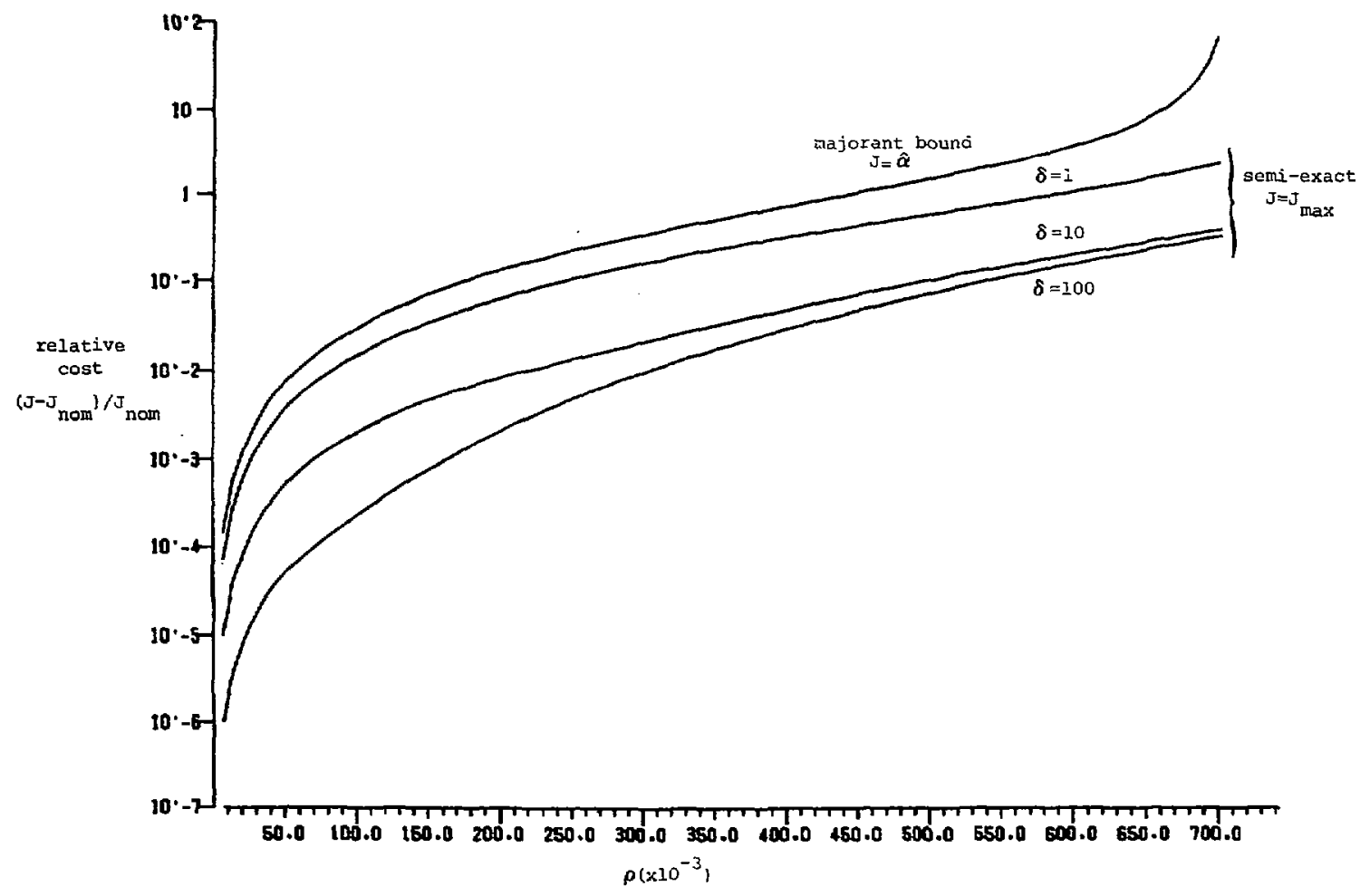

Fig. 2.

Hence, for large $\delta$ the majorant bound (6.2) is, at worst, and the system has the performance bound conservative by a factor of 2 compared to the semiexact bound.

To determine the performance bound (5.12) set $R=I_{4}$. Hence, it can be shown that

$$
\hat{\alpha}=J_{\text {nom }}+\sqrt{2}\left(\rho_{12}+\rho_{21}\right)^{2} / \nu\left(1-2 \rho_{12} \rho_{21}\right)
$$

where

$$
J_{\text {nom }}=2 / \nu
$$

$$
\rho_{12}=\gamma_{12} / \sqrt{2} \nu \hat{\delta}^{1 / 2}, \rho_{21}=\gamma_{21} / \sqrt{2} \nu \delta^{1 / 2}
$$


On the other hand, the semiexact calculation yields

$$
J_{\max }=\max _{\lambda \in[0,1)}\left\{\left[\rho_{12}^{2}+\rho_{21}^{2}+2 \rho_{12} \rho_{21} \lambda+2 \delta\left(\rho_{12} \rho_{21}\right)^{2}\left(1-\lambda^{2}\right)\right] /\right.
$$

$$
\left.\left[2 \hat{\delta}-4 \rho_{12} \rho_{21} \lambda-2 \hat{\delta}\left(\rho_{12} \rho_{21}\right)^{2}\left(1-\lambda^{2}\right)\right]\right\} .
$$

Fig. 2 compares the semiexact worst-case performance (6.8) to the majorant Lyapunov equation bound (6.7). To efficiently illustrate the results the data are specialized to the case $\rho_{12}=\rho_{21}$. Note that the semiexact performance is plotted for several values of $\delta$ because of the explicit dependence of (6.8) on $\delta$ via $\delta$.

\section{ACKNOWLEDGMENT}

The authors wish to thank J. Straehla for excellent typing of the original manuscript and $\mathrm{B}$. Bland for executing the numerical calculations.

\section{REFERENCES}

[1] M. G. Safonov and M. Athans, "Gain and phase margin for multiloop LQG regulators," IEEE Trans. Automat. Contr., vol. AC-22, pp. $173-179,1977$.

[2] M. G. Safonov, Stability and Robustness of Multivariable Feedback Systems. Cambridge, MA: M.I.T. Press, 1980.

[3] N. A. Lehtomaki, N. R. Sandell, Jr., and M. Athans, "Robustness results in linear-quadratic Gaussian based multivariable control designs," IEEE Trans. Automat. Contr., vol. AC-26, pp. 75-92, 1981.

[4] J. C. Doyle and G. Stein, "Multivariable feedback design: Concepts for a classical/modern synthesis," IEEE Trans. Automat. Contr., vol. AC-26, pp. 4-16, 1981.

[5] J. C. Doyle, "Analysis of feedback systems with structured uncertainties," IEE Proc., vol. 129 , pp. 242-250, 1982.

[6] J. C. Doyle, J. E. Wall, and G. Stein, "Performance and robustness analysis for structured uncertainty," in Proc. 21st IEEE Conf. Decision Contr., Orlando, FL, Dec. 1982, pp. 629-636.

[7] G. Zames, "Feedback and optimal sensitivity: Model reference transformations, multiplicative seminorms, and approximate in verses," IEEE Trans. Automat. Contr., vol. AC-26, pp. 301-320, 1981.

[8] G. Zames and B. A. Francis, "Feedback, minimax sensitivity, and optimal robustness," IEEE Trans, Automat. Contr., vol. AC-28, pp. $585-601,1983$.

[9] R. R. E. de Gaston and M. G. Safonov, "A homotopy method for nonconservative stability robustness analysis," in Proc. 24th IEEE Conf. Decision Contr., Fort Lauderdale, FL, Dec. 1985, pp. 12941301 .

[10] M. K. H. Fan and A. L. Tits, "Characterization and efficient computation of the structured singular value," IEEE Trans. Automat. Contr., vol. AC-31, pp. 734-743, 1986.

[11] O. D. I. Nwokah, "The quantitative design of robust multivariable control systems," in Proc. IEEE Conf. Decision Contr., Athens, Greece, Dec. 1986, pp. 16-24.

[12] I. R. Petersen and C. V. Hollot, "A Riccati equation approach to the stabilization of uncertain systems," Automatica, vol. 22, pp. 397$411,1986$.

[13] R. K. Yedavalli, S. S. Banda, and D. B. Ridgely, "Time-domain stability robustness measures for linear regulators," $J$. Guidance Contr. Dyn., vol. 8, pp. 520-524, 1985.

[14] R. K. Yedavalli, "Perturbation bounds for robust stability in linear state space models," Int. J. Contr., vol. 42, pp. 1507-1517, 1985.

[15] _ "Improved measures of stability robustness for linear state space models,"' IEEE Trans. Automat. Contr., vol. AC-30, pp. 577-579, 1985.

[16] A. R. Galimidi and B. R. Barmish, "The constrained Lyapunov problem and its application to robust output feedback stabilization," IEEE Trans. Automat. Contr., vol. AC-31, pp. 410-419, 1986.

[17] D. S. Bernstein and S. W. Greeley, "Robust output-feedback stabilization: Deterministic and stochastic perspectives," in Proc. Amer. Contr. Conf., Seattle, WA, June 1986, pp. 1818-1826.

[18] D. S. Bernstein, "Robust static and dynamic output-feedback stabilization: Deterministic and stochastic perspectives," IEEE Trans. Automat. Contr., to be published.

[19] D. S. Bernstein and D. C. Hyland, "Optimal projection for uncertain systems (OPUS): A unified theory of reduced-order, robust control design," in Large Space Structures: Dynamics and Control, S. N. Atluri and A. K. Amos, Eds. New York: Springer-Verlag, 1987.

[20] W. M. Haddad, "Robust optimal projection control-system synthesis,", Ph.D. dissertation, Dep. Mechanical Eng., Florida Inst. Technol., Melbourne, FL, Mar. 1987.

[21] A. M. Ostrowski, "On some metrical properties of operator matrices and matrices partitioned into blocks," J. Math. Anal. Appl., vol. 2, pp. $161-209,1961$.

[22] T. Strom, "On the practical application of majorants for nonlinear matrix iterations," J. Math. Anal. Appl., vol. 41, pp. 137-147, 1973.

[23] G. Dahlquist, "On matrix majorants and minorants, with applications to differential equations," Lin. Alg. Appl., vol. 52/53, pp. 199-216, 1983.

[24] S. Barnett and C. Storey, Matrix Methods in Stability Theory. New York: Barnes and Noble, 1976.

[25] J. W. Brewer, "Kronecker products and matrix calculus in system theory," IEEE Trans. Circuits Syst,, vol. CAS-25, pp. 772-781, 1978 .

[26] A. Graham, Kronecker Products and Matrix Calculus. Chichester: Ellis Horwood, 1981

[27] C. R. Rao and S. K. Mitra, Generalized Inverse of Matrices and Its Applications. New York: Wiley, 1971.

[28] G. P. H. Styan, "Hadamard products and multivariate statistical analysis," Lin. Alg. Appl., vol. 6, pp. 217-240, 1973.

[29] A. Berman and R. J. Plemmons, Nonnegative Matrices in the Mathematical Sciences. New York: Academic, 1979.

[30] D. D. Siljak, Large-Scale Dynamic Systems. Amsterdam, The Netherlands: Elsevier/North-Holland, 1978.

[31] M. Ikeda and D. D. Siljak, "Generalized decomposition of dynamic systems and vector Lyapunov functions," IEEE Trans. Automat. Contr., vol. AC-26, pp. 1118-1125, 1981.

[32] M. Djordjevic, "Stability analysis of interconnected systems with possibly unstable subsystems," Syst. Contr. Lett., vol. 3, pp. 165$169,1983$.

[33] A. A. Martynyuk, "The Lyapunov matrix-function," Nonlinear Anal. Theory, Methods, and Appl., vol. 8, pp. 1223-1226, 1984.

[34] G. H. Golub and C. F. Van Loan, Matrix Computations. Baltimore, MD: Johns Hopkins Press, 1983.

[35] T. Kato, Perturbation Theory for Linear Operators. New York: Springer-Verlag, 1980.

[36] R. K. Mehra, "Optimization of measurement schedules and sensor designs for linear dynamic systems," IEEE Trans. Automat. Contr., vol. AC-21, pp. 55-64, 1976

[37] W. M. Wonham, Linear Multivariable Control: A Geometric Approach. New York: Springer-Verlag, 1974.

[38] G. J. Butler, C. R. Johnson, and H. Wolkowicz, "Nonnegative solutions of a quadratic matrix equation arising from comparison theorems in ordinary differential equations," SIIAM J. Alg. Disc. Meth., vol. 6, pp. 47-53, 1985.

[39] H. D. Victory, Jr., "On nonnegative solutions of matrix equations," SIAM J. Alg. Discrete Meth., vol. 6, pp. 406-412, 1985.

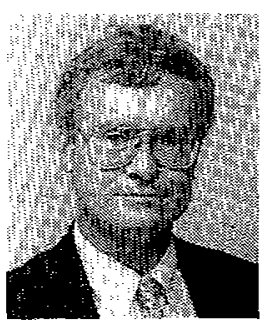

David C. Hyland received the B.S., M.S., and Sc.D. degrees in aeronautics from the Massachusetts Institute of Technology, Cambridge, in 1969,1971 , and 1973 , respectively.

After serving as a vibration specialist in a Cambridge-based acoustics consulting firm, in 1974 he joined the staff at Lincoln Laboratory, Massachusetts Institute of Technology, Cambridge. His work at Lincoln Laboratory included reentry vehicle dynamics, multibody spacecraft dynamics simulation, and spacecraft attitude control. In 1983 he joined the Government Aerospace Systems Division, Harris Corporation, Melbourne, FL, where he leads the Control Systems Analysis and Synthesis Group. His current research interests include robustness analysis for control-system design with application to vibration suppression in large flexible space structures.

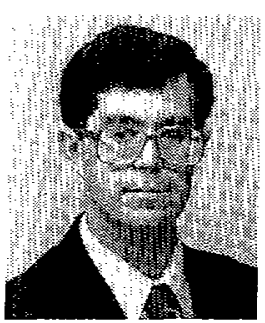

Dennis S. Bernstein (M'82) received the Sc.B. degree in applied mathematics from Brown University, Providence, RI, and the M.S.E. and $\mathrm{Ph} . \mathrm{D}$. degrees from the Computer, Information and Control Engineering Program at the University of Michigan, Ann Arbor.

After spending two years at Lincoln Laboratory, Massachusetts Institute of Technology, Lexington, he joined the Controls Analysis and Synthesis Group of the Government Aerospace Systems Division, Harris Corporation, Melbourne, FL. His current research interests include extending the optimal projection control-design approach to a variety of settings including robust, sampled-data, and decentralized control. 